\title{
Benefits and drawbacks of preimplantation genetic diagnosis (PGD) for reciprocal translocations: lessons from a prospective cohort study
}

\begin{abstract}
Paul N Scriven ${ }^{\star 1,2}$, Frances A Flinter ${ }^{1,3}$, Yakoub Khalaf ${ }^{1,4}$, Alison Lashwood ${ }^{1,3}$ and Caroline Mackie Ogilvie ${ }^{1,5}$
Preimplantation genetic diagnosis (PGD) using fluorescence in situ hybridisation probes was carried out for 59 couples carrying reciprocal translocations. Before treatment, $85 \%$ of pregnancies had resulted in spontaneous miscarriage and five couples had achieved a healthy live-birth delivery. Following treatment, $33 \%$ of pregnancies failed and 21 of 59 couples had a healthy live-born child. The accuracy of diagnosis was $92 \%$ ( $8 \%$ false abnormal and $0 \%$ false normal results). The overall incidence of 2:2 alternate segregation products was $44 \%$; however, products consistent with 2:2 adjacent segregation were $\sim$ twice as likely from male heterozygotes, and those with 3:1 disjunction were three times more likely from female heterozygotes. Our results indicate that up to three stimulation cycles per couple would give an $\sim 50 \%$ chance of a successful live birth, with the risk of miscarriage reduced to the level found in the general population. In our study, $87 \%$ of all normal/balanced embryos available were identified as being suitable for transfer. We conclude that PGD provides benefit for couples with high-risk translocations by reducing the risk of miscarriage and avoiding a pregnancy with an unbalanced form of the translocation; however, for fertile carriers of translocations with a low risk of conceiving a chromosomally unbalanced offspring, natural conception may be a
\end{abstract} more viable option.

European Journal of Human Genetics (2013) 21, 1035-1041; doi:10.1038/ejhg.2013.9; published online 6 February 2013

Keywords: PGD; FISH; reciprocal translocation; diagnostic accuracy; meiotic segregation

\section{INTRODUCTION}

Reciprocal translocations, typically an exchange of two terminal segments from different chromosomes, are found in $\sim$ one in 600 live births. ${ }^{1}$ They are associated with infertility in some carriers; for others, there may be a risk of offspring with mental and physical disability due to segregation of the translocation chromosomes at meiosis resulting in sperm or eggs with chromosome imbalance. Prenatal diagnosis can enable early diagnosis with the option of termination of pregnancy in the case of chromosome imbalance. ${ }^{2}$

Preimplantation genetic diagnosis (PGD) is used for reciprocal translocation carriers to minimise the risk of having an affected child or the distress of pregnancy termination, and to reduce the risk of miscarriage due to abnormal segregation of the translocation. ${ }^{3,4}$ Fluorescence in situ hybridisation (FISH) has been the technique most commonly used for reciprocal translocations., 5 Some approaches allow discrimination between normal and heterozygote chromosome complements, as well as detecting abnormal copy number for the translocation segments. ${ }^{7-9}$ However, the most commonly used methodology of locus-specific probes applied to interphase nuclei from cleavage stage blastomeres, ${ }^{10-16}$ or cells sampled from the trophectoderm, ${ }^{17}$ will not differentiate between normal and heterozygote chromosome complements unless probes are designed to flank closely or span the breakpoints. ${ }^{18}$

Guy's and St Thomas' Centre for PGD has undertaken 319 biopsy cycles with genetic testing for 171 couples with reciprocal translocations, which has resulted in the delivery of 85 live-born offspring. In this paper, we present an analysis of meiotic segregation modes, live-birth outcomes and referral reasons for 59 of these couples who have completed their treatment, and use these data to assess the benefit that PGD may offer to carriers of these chromosome rearrangements.

\section{MATERIALS AND METHODS}

Patients

Our prospective cohort study included the first 59 consecutive couples with reciprocal translocations referred between April 1998 and August 2003 to the Guy's and St Thomas' Centre for PGD who attempted PGD and were deemed to have completed or ceased treatment after a total of 132 stimulated cycles by March 2011. Out of the 59 couples, 55 have not had a PGD cycle in the last five years, and four have had at least two stimulated cycles and one baby. Acknowledging the significant cancellation rate for first cycles and in order to minimise potential study bias, subgroup analysis was limited to 79 stimulated cycles between April 1999 and July 2006 for the 43 couples who achieved live birth at their first attempt or were able to have a second cycle.

This report is part of a long-term study of PGD, which was approved by the Guy's and St Thomas' Hospital Ethics Committee, Lambeth, Southwark and Lewisham Health Commission (Ref: EC93/046). Written consent was obtained from the couples for testing and further study of their embryos in accordance with Human Fertilisation and Embryology Authority research licence (R0075).

Investigations for miscarriage factors were carried out where appropriate before proceeding to PGD, and included a uterine cavity assessment and

${ }^{1}$ Guy's and St Thomas' Centre for PGD, Guy's and St Thomas' Hospital NHS Foundation Trust, London, UK; ²Division of Genetics and Molecular Medicine, King's College London, School of Medicine at Guy's, King's College and St Thomas Hospitals, London, UK; ${ }^{3}$ Genetics Centre, Guy's and St Thomas' NHS Foundation Trust, London, UK; ${ }^{4}$ Assisted Conception Unit, Guy's and St Thomas' Hospital NHS Foundation Trust, London, UK; ${ }^{5}$ Cytogenetics Department, Guy's and St Thomas' NHS Foundation Trust, London, UK *Correspondence: Dr PN Scriven, Cytogenetics Department, 5th Floor Tower Wing, Guy's Hospital, Great Maze Pond, London SE1 9RT, UK. Tel: +44(0) 207 188 1705; Fax: +44(0) 207188 1697; E-mail: paul.scriven@kcl.ac.uk

Received 9 October 2012; revised 8 January 2013; accepted 10 January 2013; published online 6 February 2013 
testing for antiphospholipid antibodies. There were 37 female carriers and 22 male carriers with histories including recurrent miscarriage, infertility, a previous affected child, pregnancy termination and a history of an unbalanced translocation in the extended family (Table 1 and see Supplementary Appendix I). Before PGD, the couples had a total of 162 pregnancies: five (3\%) were live born and had a normal karyotype or the children were described to be healthy, $138(85 \%)$ resulted in spontaneous miscarriage, and the remainder were terminated or resulted in offspring with an unbalanced translocation.

\section{Assisted conception and genetic testing}

Procedures were performed as described previously. ${ }^{19,20}$ In brief, a standard long stimulation protocol for controlled ovarian stimulation was followed by IVF, or ICSI when the patient had suboptimal semen characteristics, then biopsy of one or two cells from cleavage-stage embryos 3 days after normal (2PN) fertilisation for testing, followed by embryo transfer on day 4 (included in the subgroup analysis) and day 5 (the most recent cycles and not included in the subgroup analysis). A maximum of three embryos were transferred. A one-cell biopsy strategy was used for 58/59 couples. Blastomeres and whole embryos were spread using the Tween/ $\mathrm{HCl}$ method. ${ }^{21}$ For confirmation of diagnosis, at least two nuclei with the same deviant single probe signal or two deviant signals consistent with plausible mitotic mechanisms, such as chromosome nondisjunction and anaphase lag were determined to be true findings. On the basis of the analysis of all the cells from the spread embryo, the potential source of the error causing the false abnormal index results was differentiated into that owing to the limitations of the FISH technique and that due to mosaicism. Deviant results consistent with mitotic nondisjunction or anaphase lag were assigned as mosaicism, and results that were not consistent with the likely meiotic segregation mode of the translocation and a plausible postzygotic mitotic mechanism were assigned as FISH error. Where it was not possible to differentiate the type of error (eg, absence of a single probe signal in one nucleus for a chromosome with only one diagnostic probe, which could be consistent with postzygotic loss of the chromosome or failure to detect two signals due to signal colocalisation or localised binding failure of the FISH probe), false abnormal results were assigned to be due to 'mosaicism or FISH'.

Individual probe sets, usually comprising commercially available chromosome subtelomere and centromere probes, were designed for each couple, and tested on metaphase and interphase cells from peripheral lymphocytes of both partners, as part of their precycle workup. FISH probes were directly or indirectly labelled and sourced from different manufacturers (see Supplementary Appendix II). The choice of probes was informed by the predicted most frequent products leading to chromosome imbalance, and any products likely to result in live-born offspring with chromosome imbalance. ${ }^{5}$ The risk of clinically significant misdiagnosis (a viable unbalanced translocation product) was minimised either by using two diagnostic probes for potentially viable imbalance, or by testing two cells and transferring embryos with concordant normal/balanced results from both cells.

The likely mode of segregation based on segment copy number was elucidated as described previously. ${ }^{22}$ For the diagnostic accuracy part of the study, embryos were spread on day 4 and confirmed, using the same test as before, to be normal/balanced if at least $50 \%$ of nuclei were consistent with normal copy number for the chromosomes tested. ${ }^{23}$ Abnormal test results were deviations from a normal test result and spread embryos were confirmed to be abnormal if greater than $50 \%$ of nuclei were abnormal, and assigned to be consistent with 2:2 adjacent-1,2:2 adjacent-2, 3:1 or 4:0 disjunction at meiosis if at least two nuclei obtained showed the appropriate and consistent deviation from two signals for each chromosome region tested. Haploid and triploid results were differentiated from 4:0 segregation products by retesting with a probe specific for a chromosome not involved in the translocation. The likely segregation mode was deemed to be unknown if these criteria were not met.

\section{Statistical analysis}

The live birth rate per couple was calculated from 132 stimulated cycles, and also estimated with the assumption that unsuccessful couples who did not return for subsequent cycles would have had the same chance of success as those couples who continued treatment. Pregnancy rates per cycle were calculated to allow comparison with other studies.

For the diagnostic accuracy part of the study, biopsy (index) results with an unknown outcome (reference standard) were initially allocated in proportion to normal and abnormal biopsy results with a known outcome because diagnostic accuracy measures are sensitive to prevalence. There were no known false normal index results; however, a significant proportion of embryos with a normal biopsy result were transferred without success and lost to follow-up. Therefore, in a sensitivity analysis, we varied the allocation of normal biopsy results up to the upper $95 \%$ confidence limit of zero. ${ }^{23}$ Diagnostic accuracy measures calculated were: false positive and false negative (incorrect abnormal and normal biopsy results calculated as the proportion of the total outcomes), overall accuracy (the proportion of all biopsy results that were correct), sensitivity (the proportion of abnormal embryos that had an abnormal biopsy result) and specificity (the proportion of normal embryos that had a normal biopsy result), the positive predictive value (the proportion of abnormal biopsy results that were correct) and, most relevant to clinical misdiagnosis, the negative predictive value (the proportion of normal biopsy results that were correct)

For study measures, 95\% confidence intervals (CIs) were calculated to indicate precision. Pearson's goodness of fit $\chi^{2}$ or Fisher's exact test was used to calculate significance probabilities. Logistic regression and the odds ratio (OR) were used to compare predictors.

\section{RESULTS}

\section{PGD cycles and clinical outcome}

Figure 1 shows the study flow chart. Cycle and clinical outcome details are presented in Table 2 and see Supplementary Appendix III. Out of 59 couples completing PGD, 28 couples had at least one pregnancy $(47 \%, 95 \%$ CI $34-61 \%), 21$ couples $(36 \%, 95 \%$

Table 1 Referral indications for the 59 carriers of reciprocal translocations

\begin{tabular}{|c|c|c|c|c|c|}
\hline Referral indication & Female carrier (\%) & Live-birth risk (\%) & Male carrier (\%) & Live-birth risk (\%) & Total (\%) \\
\hline Affected child & $4(11)$ & $3.7-20$ & - & - & $4(7)$ \\
\hline Affected child and recurrent miscarriage & $2(5)$ & $15-20$ & $1(5)$ & 35 & $3(5)$ \\
\hline Affected stillbirth & $1(3)$ & 1 & - & - & $1(2)$ \\
\hline Family history & $1(3)$ & 20 & - & - & $1(2)$ \\
\hline Female infertility & $3(8)$ & $1-3.7$ & $1(5)$ & $5-10$ & $4(7)$ \\
\hline Male infertility & - & - & $12(55)$ & $0-1$ & $12(20)$ \\
\hline Male infertility and ICSI recurrent miscarriage & - & - & $1(5)$ & $<1$ & $1(2)$ \\
\hline Termination & $3(8)$ & $5-7$ & $1(5)$ & 10 & $4(7)$ \\
\hline Termination and recurrent miscarriage & $2(5)$ & $3.7-10$ & - & - & $2(3)$ \\
\hline Termination and secondary infertility & - & - & $1(5)$ & 5 & $1(2)$ \\
\hline Recurrent miscarriage & $21(57)$ & $<1-5$ to 10 & $5(23)$ & $<0.7-20$ & $26(44)$ \\
\hline Total & 37 & $<1-20$ & 22 & $<1-35$ & 59 \\
\hline
\end{tabular}




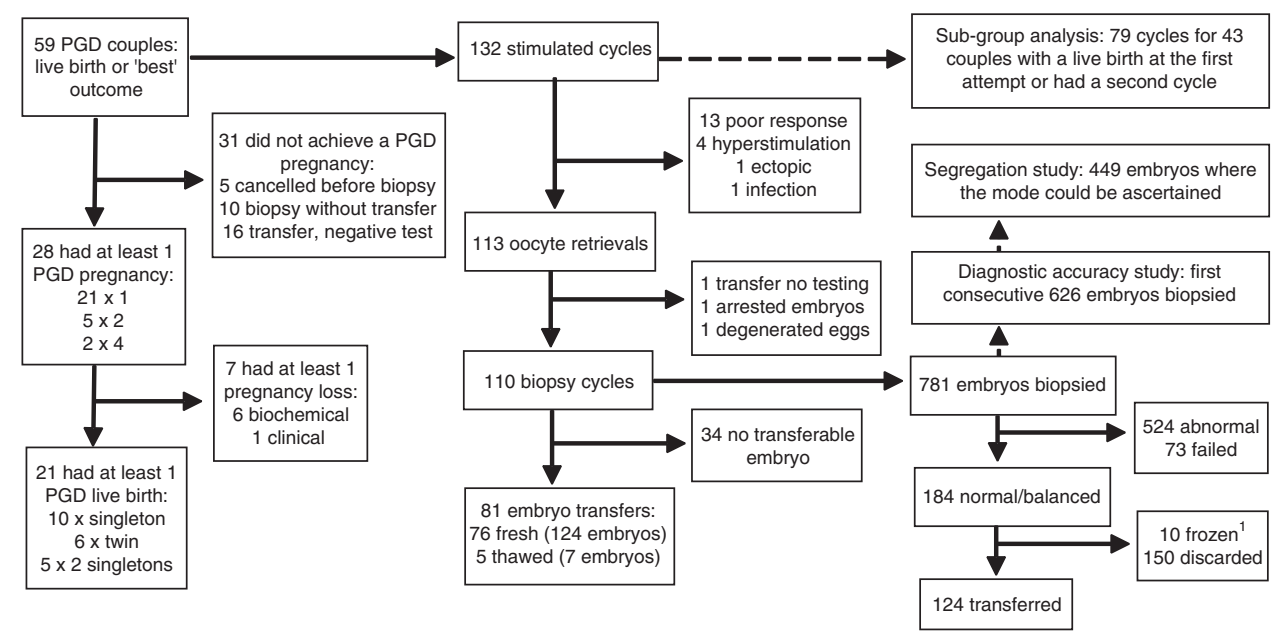

Figure 1 Study flow chart.

${ }^{1}$ Subsequently thawed and seven transferred and three discarded.

\section{Table 2 Patient and cycle summary}

\begin{tabular}{|c|c|}
\hline Couples started & 59 \\
\hline Mean maternal age, first cycle, years $\pm S D$ (range) & $34.3 \pm 4.0(23-42)$ \\
\hline Stimulated cycles (cycles started) (CS) & 132 \\
\hline Mean maternal age \pm SD (range) & $34.7 \pm 3.9(23-43)$ \\
\hline Cycles cancelled before oocyte retrieval (OR) $(\%)$ & 19 (14) \\
\hline Cycles to OR (\%) & $113(86)$ \\
\hline Median number of oocytes retrieved (range) & $13(3-39)$ \\
\hline Median number of 2PN zygotes (range) & $8(1-20)$ \\
\hline Cycles cancelled after oocyte retrieval ${ }^{b}$ & 3 \\
\hline Cycles to biopsy (\%) & $110(83)$ \\
\hline Embryos tested on day 3 ; median (range) & $781 ; 6(1-20)$ \\
\hline Normal; abnormal; failed ${ }^{\mathrm{c}}(\%)$ & 184 (24); 524 (67): 73 (9) \\
\hline Transferable results (TR) $(\%, 95 \% \mathrm{Cl})$; median (range) & $184(24,21-27) ; 1(0-7)$ \\
\hline Couples with a fresh embryo transfer (\%) & $44(75)$ \\
\hline Fresh embryo transfers (ET) (\%) & $76(56)$ \\
\hline Couples with a frozen embryo transfer (\%) & $4(7)$ \\
\hline Frozen embryo transfers (FET) & 5 \\
\hline Fresh embryos transferred; median (range) & $124 ; 2(1-3)$ \\
\hline Fresh embryos transferred per transfer: $1,2,3(\%)$ & $30(39), 44(58), 2$ (3) \\
\hline Thawed embryos transferred (range) & $7(1-2)$ \\
\hline Fresh hCG positive pregnancies ( $\%$ per CS, OR, ET) & $37(28,33,49)$ \\
\hline Frozen hCG positive pregnancies (\% per FET) ${ }^{\mathrm{d}}$ & $2(10)$ \\
\hline FHB-positive pregnancies ( $\%$ per CS, OR, ET) & $29(22,26,38)$ \\
\hline Foetal sacs; heart beats (implantation rate \%) & $37(30) ; 36(29)$ \\
\hline Couples successful at attempt $1,2,3,4,5,6$ & $8,11,2,0,0,0$ \\
\hline Live-birth pregnancies ( $\%$ per CS, $95 \% \mathrm{CI}$ ) & $26(20,13-28)$ \\
\hline Couples with at least one live-birth pregnancy $(\%, 95 \% \mathrm{Cl})$ & $21(36,24-49)$ \\
\hline Pregnancy losses ( $\%$ per hCG + ve pregnancy, $95 \% \mathrm{Cl})^{\mathrm{e}}$ & $13(33,19-50)$ \\
\hline Couples with at least one pregnancy $(\%, 95 \% \mathrm{Cl})$ & $28(47,34-61)$ \\
\hline Couples with at least one pregnancy loss $(\%, 95 \% \mathrm{Cl})$ & $10(36,19-56)$ \\
\hline Live-born offspring; singleton; twin deliveries (\%) & $32 ; 20(77) ; 6(23)$ \\
\hline $46, X Y$; heterozygote male; male not karyotyped ${ }^{f}$ & \\
\hline $46, \mathrm{XX}$; heterozygote female; female not karyotyp & \\
\hline
\end{tabular}

aThirteen cycles cancelled due to poor response, four cycles due to hyperstimulation risk, one cycle due to spontaneous ectopic pregnancy detected at down regulation, one cycle due to Escherichia coli infection.

${ }^{5}$ One cycle with only three 2PN embryos had transfer without testing, one cycle cancelled due to arrested embryos, one cycle had degenerated eggs.

cNo FISH result, associated with a highly fragmented nucleus, a cell without a nucleus from an embryo with only five cells, or no hybridisation.

${ }^{\mathrm{d} B i o c h e m i c a l}$ only pregnancies.

eNine biochemical-only pregnancies (including two FET); 15 weeks gestation missed abortion, products not karyotyped due to formalin fixation; 1 12weeks gestation miscarriage (the couple declined to have the products karyotyped); one twin pregnancy at 19 weeks gestation due to infection (a balanced male heterozygote and a female normal for chromosome 13, 18, 21 and $X X$ (partial PCR result only)); one termination of pregnancy at 10 weeks gestation $(5.6 \mathrm{~mm}$ NT and hydrops and a 47,XY, +18 karyotype detected following CVS).

fOne 25 weeks twin delivery following spontaneous rupture of membranes at 18 weeks; one twin died at $48 \mathrm{~h}$ and was not karyotyped.

CI 24-49\%) had at least one live birth and 10 couples (36\%, 95\% CI $19-56 \%)$ had at least one pregnancy loss. The median time from referral to first delivery was 23 months (range 17-44 months).

The estimated live birth rate per couple, assuming that unsuccessful couples who did not return for subsequent treatment cycles would have had the same chance of success as those who did, was 30/59 (51\%, 95\% CI 38-64\%) after three to six cycles (see Supplementary Appendix IV), with a theoretical total of 145-232 cycles.

The average age of the female partners at the first cycle attempt was 34 years (range 26-42 years) and the age of the oldest successful female partner was 41 years. In our subgroup analysis for every 1 year increase in age, the odds of a live-birth pregnancy decreased by a factor of only $\sim 2 \%$ (OR $0.98,95 \%$ CI $0.86-1.11, P=0.724$ ), and the date of stimulation was not an important predictor of a live birth (OR 0.86, 95\% CI 0.64-1.15, $P=0.314$ ). No difference was found in the live birth or miscarriage rates between male and female carriers, fertile and infertile couples, or between couples with three or more miscarriages and those with fewer miscarriages (see Supplementary Appendix V).

The median number of eggs collected was 13 (range 4-39) and the median number of embryos biopsied was 6 (range 1-20). In our study the number of oocytes was not an important predictor for a live-birth pregnancy (OR 1.0, 95\% CI 0.92-1.10, $P=0.960$ ). For each additional embryo biopsied, the odds of a live birth increased by a factor of $\sim 13 \%$ (OR 1.13, 95\% CI 0.98-1.30, $P=0.082$ ).

A total of $29 / 79$ (37\%) cycles had no embryo available for transfer and 50/79 (63\%) stimulated cycles had at least one embryo available for transfer. Biopsy cycles with more than one transferable embryo after testing were $\sim$ eight times more likely to achieve a live birth than those with only one embryo available for transfer (OR 8.49, 95\% CI 1.62-44.44, $P=0.011)$. Cycles with more than one embryo transferred were $\sim$ five times more likely to achieve a live birth than those with only one embryo transferred (OR 5.38, 95\% CI 1.26-23.0, $P=0.023$ ). Theoretically, the probability of transferring a diploid embryo without aneuploidy for a nontranslocation chromosome is higher if more than one embryo is transferred. Although, not statistically significant, our results suggest that the odds of a spontaneous miscarriage following PGD might be reduced by transferring more than one embryo (OR $0.23,95 \%$ CI $0.03-1.28, P=0.248$ ). However, of 17 pregnancies resulting from the transfer of more than one embryo, seven $(41 \%$, 95\% CI 18-67\%) resulted in a multiple (twin) pregnancies and two of those had foetal complications.

\section{Diagnostic accuracy}

Of the first consecutive 626 embryos tested, 558 (89\%) embryos had a successful biopsy result and 68 (11\%) failed; 137 (22\%) embryos had 
a normal result and $421(67 \%)$ an abnormal result. Out of 506 embryos spread on day 4 for COD, 114 (22.5\%) were mosaic with evidence of two or more normal/balanced or unbalanced cell lines, of which 22 (19\%) had a normal/balanced cell line and 92 (81\%) had only unbalanced cell lines (see Supplementary Appendix VI).

All $24(100 \%)$ spread embryos and 26 offspring with a normal biopsy (index) result were confirmed to have a normal/balanced complement for the chromosomes tested (reference standard); 87 embryos that did not have a confirmation result were therefore initially assigned as normal. Out of 367 embryos with an abnormal index result and a known outcome, 327 (89\%) were confirmed to be abnormal, and 40 were normal on follow-up. The 54 embryos that did not have a confirmation result were therefore apportioned as 48 abnormal and six normal. Out of 558 successful index results (see Supplementary Appendix VII) there were 46 (8\%) false abnormal results and no false normal results. The test accuracy was estimated to be $92 \%(512 / 558)$ with $100 \%$ (375/375) sensitivity and $75 \%$ (137/ $183)$ specificity. With $67 \%$ (375/558) prevalence, the predictive value was estimated to be $89 \%$ (375/421) for an abnormal test result and $100 \%(137 / 137)$ for a normal test result. Following sensitivity analysis, the prevalence changed to $68 \%(379 / 558)$ and the sensitivity and negative predictive value to $99 \%(375 / 379)$ and $97 \%(133 / 137)$, respectively.

There were 40 false abnormal index results before apportioning the unknown outcome results. Analysis of the deviant signal patterns of these embryos allowed tentative assignment of the cause of error: $25 \%$ (5/20) due to FISH and 75\% (15/20) due to mosaicism; for the remaining 20 embryos it was not possible to differentiate between these causes of error.

Out of the first 626 embryos tested, a total of 74 potentially transferable embryos were excluded ( 28 due to failure to obtain a diagnosis and 46 due to a false abnormal test result), which was $12 \%$ (74/626) of the total embryos and 35\% (74/211) of the normal/ balanced embryos. Out of these 74 embryos, $56(75 \%)$ were likely to have been excluded due to mosaicism and 18 (25\%) due to the limitations of the FISH technique. If a genetic test with $100 \%$ accuracy had been used, 25\% $((137+18) / 626)$ of all embryos would have been available for transfer, which compares with $22 \%(137 / 626)$ obtained using FISH (difference 13\%, $P=0.229$ ).

\section{Meiotic segregation products and putative mosaicism aetiology}

A mode of segregation consistent with the chromosome complement was elucidated for 449 embryos (Figure 2 and see Supplementary Appendix VIII); 44\% were consistent with 2:2 alternate segregation resulting in a normal or balanced chromosome complement, and

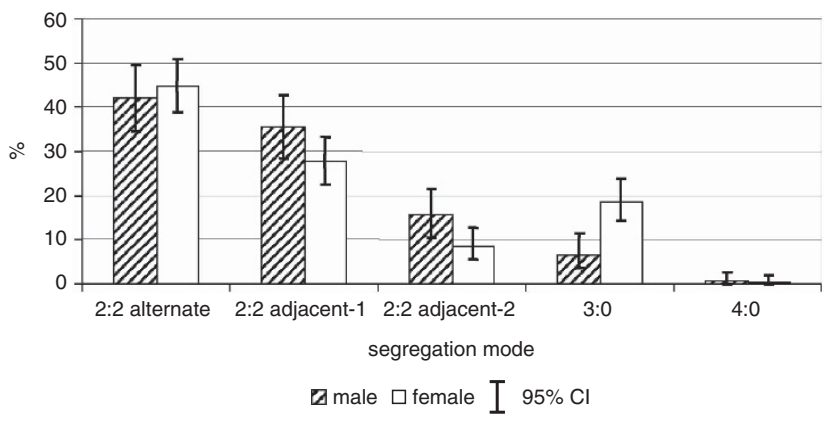

Figure 2 Embryo chromosome complements consistent with reciprocal translocation meiotic segregation.
$56 \%$ were consistent with other modes resulting in an unbalanced translocation chromosome complement. Female and male heterozygotes had a similar proportion of embryos that were consistent with 2:2 alternate segregation; however, male heterozygotes were $\sim$ two times more likely to have embryos consistent with 2:2 adjacent segregation (OR 1.82, 95\% CI 1.24-2.67, $P=0.002$ ) and female heterozygotes $\sim$ three times more likely to have embryos consistent with $3: 1$ disjunction (OR 3.23, 95\% CI 1.67-6.26, $P=0.0003$ ).

Fifty two embryos had at least one deviant nonpolyploid nucleus with an interpretable signal pattern, of which 48 (92\%) had monosomy for one or more chromosomes consistent with anaphase lag, three $(6 \%)$ were consistent with nondisjunction resulting in complementing monosomy and trisomy cell lines, and one (2\%) was consistent with a terminal deletion (see Supplementary Appendix IX).

\section{DISCUSSION}

This study has confirmed that reciprocal translocation carriers produce a large number of abnormal gametes, and we have refined with greater confidence the observations and conclusions in previous reports, indicating that the incidence of 3:1 disjunction is likely to be greater for female heterozygotes and 2:2 adjacent segregation to be higher for male carriers. ${ }^{22,24-26}$ It is therefore particularly important that any diagnostic technique has low failure and high accuracy with as few false normal and abnormal results as practicable. In addition to the limitations of any genetic testing technology, mosaicism will also lead to inaccurate results although, as seen in our study and others, ${ }^{23,27}$ the majority of mosaic embryos have only abnormal cells, which mitigates the extent of the error. ${ }^{28}$ Our study also confirms that mosaicism resulting from mitotic anaphase lag is the predominant plausible mechanism. ${ }^{29}$

Data from multiple centres carrying out PGD are collected by the ESHRE PGD Consortium; ${ }^{6}$ these data for reciprocal translocations and for three large single-centre studies ${ }^{24,30,31}$ are shown in Table 3. The overall pregnancy and live birth rates for our couples are slightly higher than in these previous studies. In our study, two embryos were transferred when available and there was a strong association between live birth following PGD and the number of embryos available for transfer, and the number of embryos transferred. However, of seven twin pregnancies, two had serious complications and it is important to consider the implications of establishing a multiple pregnancy, including a significantly increased risk of premature delivery and neonatal complications, which may be permanent. ${ }^{32}$ Our current policy is to strongly encourage couples to accept single embryo transfer where the female partner is 35 years or under.

Some researchers have suggested that the effectiveness of PGD should be measured by comparing the outcome for couples after treatment with their history before treatment. ${ }^{33,34}$ However, others have acknowledged that this approach is likely to be biased because translocation couples with unfortunate reproductive histories are more likely to be referred for PGD than couples who do not have exceptional histories. ${ }^{30}$ It is also worth noting that addressing other factors contributing to the risk of spontaneous miscarriage is likely to improve the outcome without PGD. ${ }^{35}$

Table 1 describes the reproductive histories for the couples in this study. It is interesting to note that the individual translocations carried by these couples have risks for live birth with chromosomal imbalance ranging from $1-35 \%$, and, as expected, the higher-risk translocations were found in the couples presenting for PGD because of a previous live-born child with genetic imbalance. PGD is therefore clearly beneficial for this group, giving a substantial reduction in the risk of an affected pregnancy with an unbalanced translocation, and a 
Table 3 Comparison of outcome measures for several larger studies

\begin{tabular}{|c|c|c|c|c|c|c|c|c|c|c|}
\hline \multirow[b]{2}{*}{ Outcome measure per cycle } & \multicolumn{2}{|c|}{ Present study } & \multicolumn{2}{|c|}{ Fischer et al. ${ }^{30}$} & \multicolumn{2}{|c|}{ Keymolen et al. ${ }^{31}$} & \multicolumn{2}{|c|}{ Ko et al. ${ }^{24}$} & \multicolumn{2}{|c|}{ Harper et al. ${ }^{6}$} \\
\hline & Calculated & $\%(95 \% \mathrm{Cl})$ & Calculated & $\%(95 \% \mathrm{Cl})$ & Calculated & $\%(95 \% \mathrm{Cl})$ & Calculated & $\%(95 \% \mathrm{Cl})$ & Calculated & $\%(95 \% \mathrm{Cl})$ \\
\hline Oocyte retrieval & $26 / 113$ & $23(16-32)$ & - & - & $40 / 312$ & $13(9-17)$ & - & - & $60 / 436$ & $14(11-17)$ \\
\hline Biopsy & $26 / 110$ & $24(16-33)$ & - & - & - & - & - & - & $60 / 418$ & $14(11-18)$ \\
\hline Embryo transfer & $26 / 81$ & $32(22-43)$ & - & - & $40 / 151$ & $26(20-34)$ & $24 / 116$ & $21(14-29)$ & $60 / 247$ & $24(19-30)$ \\
\hline Biopsy & $26 / 110$ & $24(16-33)$ & $43 / 220$ & $20(15-25)$ & - & - & - & - & - & - \\
\hline Embryo transfer & $26 / 81$ & $32(22-43)$ & $43 / 131$ & $33(25-42)$ & - & - & - & - & - & - \\
\hline \multicolumn{11}{|l|}{ Clinical pregnancies (FHB): } \\
\hline Oocyte retrieval & $29 / 113$ & $26(18-35)$ & - & - & $40 / 312$ & $13(9-17)$ & - & - & $341 / 2413$ & $14(13-16)$ \\
\hline Biopsy & 29/110 & $26(18-36)$ & - & - & - & - & - & - & $341 / 2250$ & $15(14-17)$ \\
\hline
\end{tabular}

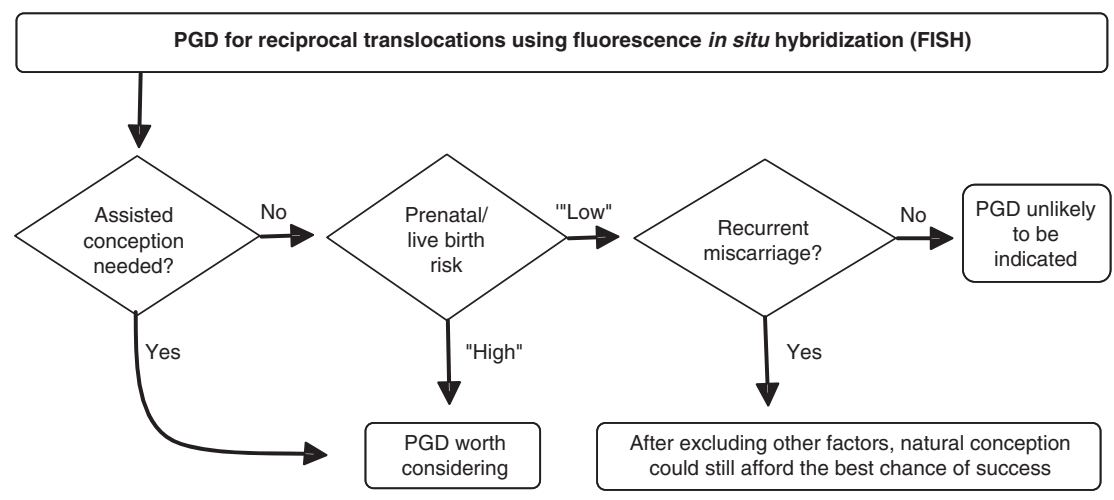

Figure 3 Pathways to PGD for reciprocal translocations using FISH.

reduction in the risk of miscarriage. For female carriers presenting only with recurrent miscarriages, however, the risk associated with their translocations is lower (max 10\%). Franssen et al. ${ }^{36}$ compared the reproductive outcome without PGD intervention in a two year period for 157 couples with reciprocal translocations and a history of at least two miscarriages and 409 couples with normal karyotypes. Over the study period, the live birth rate was broadly similar between the two groups ( $83 \%$ for translocation couples and $84 \%$ for couples with normal karyotypes). In comparison, our experience of PGD for reciprocal translocation couples indicates that over a similar time period, $51 \%$ of couples might expect to be successful following PGD; PGD could therefore be potentially reducing the chances of a live birth for fertile couples. Furthermore, couples committing to PGD are required to have protected sexual intercourse to preclude spontaneous pregnancy during treatment, and it could take up to 4 years to achieve a live birth. Our experience of timescale appears to be broadly similar to those reported by other researchers for chromosome rearrangements, which have included 3 months engaged in ART (an average of 1.4 cycles) for the first delivery ${ }^{30}$ and a median of 15.5 months (range 9-76 months) between completion of the PGD workup and first delivery. ${ }^{31}$

Figure 3 shows an outline decision pathway for PGD using FISH and day 3 biopsy for reciprocal translocation couples. From our study we conclude that for infertile couples the incidence of chromosome abnormality in their embryos is likely to be high and therefore PGD is worth considering, because a well designed test for the translocation has high predictive value. For couples who do not need assisted conception, natural conception might afford the best chance of having a healthy child. However, for couples with a high-risk translocation, the relatively low pregnancy rate associated with PGD might be of secondary concern to the risk of an affected pregnancy.

Is there potential for improving the live birth rates for these couples? This might be achieved by more accurate technologies, thus reducing the false abnormal rate, and increasing the number of embryos available for transfer. PGD using PCR microsatellite analysis, with the facility to detect clinically relevant uniparental disomy, may offer the promise of some improvement over the FISH technique. ${ }^{37,38}$ Array comparative genomic hybridisation and single nucleotide polymorphism microarray provide copy number information for every other chromosome in addition to those involved in the chromosome rearrangement. ${ }^{39-42}$ However, these findings will reduce the number of embryos available for transfer by detecting abnormalities, which may not persist during embryo development, or may be clinically insignificant. The data we present here demonstrate that reciprocal translocation couples are likely to have relatively few normal/balanced embryos; rejecting embryos with apparent 
aneuploidy, for chromosomes not involved in the translocation can only reduce this already small cohort. Most centres screening for preimplantation aneuploidy now recognise that cleavage stage is not an appropriate testing point, due to the high levels of mosaicism. Any move to use whole chromosome screening for reciprocal translocation couples would require testing blastocyst biopsies rather than cleavage-stage embryos.

In summary, our study suggests that in experienced hands, the chance of a live-birth delivery following PGD and testing two chromosome pairs using the FISH technique is likely to be around $20 \%$ per stimulated cycle and up to $50 \%$ per couple if three cycles are possible. The risk of spontaneous miscarriage following PGD for the translocation is likely to be reduced at least to that observed in the general population. However, couples should expect that the duration of the entire PGD treatment from referral to first live-birth delivery could be up to 4 years, in which time more than one successful natural conception could be carried to term for fertile carriers of lowrisk translocations. We conclude that PGD provides benefit for couples with high-risk translocations by reducing the risk of miscarriage and avoiding a child with an unbalanced form of the translocation. However, for fertile carriers of translocations with a low risk of unbalanced offspring, the drawbacks of PGD include the cost, extended time frame and relatively low success rate for the process; therefore, natural conception may be a more viable option, and the 'technological imperative' and patient expectations need to be managed appropriately by the geneticists and assisted conception personnel who counsel the patients.

\section{CONFLICT OF INTEREST}

The authors declare no conflict of interest.

\section{ACKNOWLEDGEMENTS}

Toby Kirby provided first-class technical expertise during the course of this study. We are also grateful to Peter Braude for his advice and guidance throughout this period, and to our many colleagues, past and present, for their contributions to the success of our PGD programme for couples with chromosome rearrangements.

1 Jacobs PA, Browne C, Gregson N, Joyce C, White H: Estimates of the frequency of chromosome abnormalities detectable in unselected newborns using moderate levels of banding. J Med Genet 1992; 29: 103-108.

2 Gardner RJM, Sutherland GR, Shaffer LG: Chromosome Abnormalities and Genetic Counseling, 4th Ed New York, USA: Oxford University Press, 2012.

3 Munné S: Preimplantation genetic diagnosis of numerical and structural chromosome abnormalities. Reprod Biomed Online 2002; 4: 183-196.

4 Ogilvie CM, Braude PR, Scriven PN: Preimplantation genetic diagnosis-an overview. J Histochem Cytochem 2005; 53: 255-260.

5 Scriven PN, Handyside AH, Ogilvie CM: Chromosome translocations: segregation modes and strategies for preimplantation genetic diagnosis. Prenat Diagn 1998; 18 1437-1449.

6 Harper JC, Coonen E, De Rycke M et al: ESHRE PGD Consortium data collection X: cycles from January to December 2007 with pregnancy follow-up to October 2008. Hum Reprod 2010; 25: 2685-2707.

7 Munné S, Morrison L, Fung J et al: Spontaneous abortions are reduced after preconception diagnosis of translocations. J Assist Reprod Genet 1998; 15: 290-296.

8 Willadsen S, Levron J, Munne S et al: Rapid visualization of metaphase chromosomes in single human blastomeres after fusion with in-vitro matured bovine eggs. Hum Reprod 1999; 14: 470-475.

9 Verlinsky Y, Cieslak J, Evsikov S, Galat V, Kuliev A: Nuclear transfer for full karyotyping and preimplantation diagnosis for translocations. Reprod Biomed Online 2002; 5: 300-305.

10 Munné S, Fung J, Cassel MJ, Márquez C, Weier HU: Preimplantation genetic analysis of translocations: case-specific probes for interphase cell analysis. Hum Genet 1998 102: 663-674.

11 Pierce KE, Fitzgerald LM, Seibel MM, Zilberstein M: Preimplantation genetic diagnosis of chromosome balance in embryos from a patient with a balanced reciprocal translocation. Mol Hum Reprod 1998; 4: 167-172.
12 Munné S, Sandalinas M, Escudero T, Fung J, Gianaroli L, Cohen J: Outcome of preimplantation genetic diagnosis of translocations. Fertil Steril 2000; 73: 1209-1218.

13 Fridstrom M, Ahrlund-Richter L, Iwarsson E et al: Clinical outcome of treatment cycles using preimplantation genetic diagnosis for structural chromosomal abnormalities. Prenat Diagn 2001; 21: 781-787.

14 Ogilvie CM, Braude P, Scriven PN: Successful pregnancy outcomes after preimplantation genetic diagnosis (PGD) for carriers of chromosome translocations. Hum Fertil 2001; 4: 168-171.

15 Simopoulou M, Harper JC, Fragouli E et al: Preimplantation genetic diagnosis of chromosome abnormalities: implications from the outcome for couples with chromosomal rearrangements. Prenat Diagn 2003; 23: 652-662.

$16 \mathrm{Lim}$ CK, Jun JH, Min DM et al: Efficacy and clinical outcome of preimplantation genetic diagnosis using FISH for couples of reciprocal and Robertsonian translocations: the Korean experience. Prenat Diagn 2004; 24: 556-561.

17 de Boer KA, Catt JW, Jansen RP, Leigh D, McArthur S: Moving to blastocyst biopsy for preimplantation genetic diagnosis and single embryo transfer at Sydney IVF. Fertil Steril 2004; 82: 295-298.

18 Weier HU, Munne S, Fung J: Patient-specific probes for preimplantation genetic diagnosis of structural and numerical aberrations in interphase cells. J Assist Reprod Genet 1999; 16: 182-191.

19 Pickering S, Polidoropoulos N, Caller J, Scriven P, Ogilvie CM, Braude PPreimplantation Genetic Diagnosis Study Group. Strategies and outcomes of the first 100 cycles of preimplantation genetic diagnosis at the Guy's and St. Thomas' Center. Fertil Steril 2003; 79: 81-90.

20 Scriven PN, Kirby TL, Ogilvie CM: FISH for pre-implantation genetic diagnosis. $J$ Vis Exp 2011; 48: e2570; doi:10.3791/2570.

21 Coonen E, Dumoulin JC, Ramaekers FC, Hopman AH: Optimal preparation of preimplantation embryo interphase nuclei for analysis by fluorescence in-situ hybridization. Hum Reprod 1994; 9: 533-537.

22 Mackie Ogilvie C, Scriven PN: Meiotic outcomes in reciprocal translocation carriers ascertained in 3-day human embryos. Eur J Hum Genet 2002; 10: 801-806.

23 Bint SM, Ogilvie CM, Flinter FA, Khalaf Y, Scriven PN: Meiotic segregation of Robertsonian translocations ascertained in cleavage-stage embryos-implications for preimplantation genetic diagnosis. Hum Reprod 2011; 26: 1575-1584.

24 Ko DS, Cho JW, Park SY et al: Clinical outcomes of preimplantation genetic diagnosis (PGD) and analysis of meiotic segregation modes in reciprocal translocation carriers. Am J Med Genet A 2010; 152A: 1428-1433.

25 Lim CK, Cho JW, Song IO, Kang IS, Yoon YD, Jun JH: Estimation of chromosomal imbalances in preimplantation embryos from preimplantation genetic diagnosis cycles of reciprocal translocations with or without acrocentric chromosomes. Fertil Steril 2008; 90: 2144-2151.

26 Ye Y, Qian Y, Xu C, Jin F: Meiotic segregation analysis of embryos from reciprocal translocation carriers in PGD cycles. Reprod Biomed Online 2012; 24: 83-90.

27 Colls $P$, Escudero $T$, Zheng $X$ et al: Increased efficiency of preimplantation genetic diagnosis for infertility through reanalysis of dubious signals. Fertil Steril 2007; 88 : 53-61.

28 Munné S, Wells D, Cohen J: Technology requirements for preimplantation genetic diagnosis to improve assisted reproduction outcomes. Fertil Steril 2010; 94: 408-430.

29 Coonen E, Derhaag JG, Dumoulin JC et al: Anaphase lagging mainly explains chromosomal mosaicism in human preimplantation embryos. Hum Reprod 2004; 19: 316-324.

30 Fischer J, Colls P, Escudero T, Munné S: 2010 Preimplantation genetic diagnosis (PGD) improves pregnancy outcome for translocation carriers with a history of recurrent losses. Fertil Steril 2010; 94: 283-289.

31 Keymolen K, Staessen C, Verpoest W, Liebaers I, Bonduelle M: Preimplantation genetic diagnosis in female and male carriers of reciprocal translocations: clinical outcome until delivery of 312 cycles. Eur J Hum Genet 2012; 20: 376-380.

32 Braude P: One Child at a Time. Reducing Multiple Births after IVF. Report of the Expert Group on Multiple Births after IVF 2006; http://www.hfea.gov.uk/docs/MBSET report.pdf.

33 Otani T, Roche M, Mizuike M, Colls P, Escudero T, Munne S: Preimplantation genetic diagnosis significantly improves the pregnancy outcome of translocation carriers with a history of recurrent miscarriage and unsuccessful pregnancies. Reprod Biomed Online 2006; 13: 869-874.

34 Verlinsky Y, Cohen J, Munne S et al: Over a decade of experience with preimplantation genetic diagnosis. Fertil Steril 2004; 82: 302-303.

35 Stephenson MD, Sierra S: Reproductive outcomes in recurrent pregnancy loss associated with a parental carrier of a structural chromosome rearrangement. Hum Reprod 2006; 21: 1076-1082.

36 Franssen MT, Korevaar JC, van der Veen F, Leschot NJ, Bossuyt PM, Goddijn M: Reproductive outcome after chromosome analysis in couples with two or more miscarriages: index [corrected]-control study. BMJ 2006; 332: 759-763.

37 Fiorentino F, Kokkali G, Biricik A et al: Polymerase chain reaction-based detection of chromosomal imbalances on embryos: the evolution of preimplantation genetic diagnosis for chromosomal translocations. Fertil Steril 2010; 94: 2001-2011.

38 Traversa MV, Carey L, Leigh D: A molecular strategy for routine preimplantation genetic diagnosis in both reciprocal and Robertsonian translocation carriers. Mol Hum Reprod 2010; 16: 329-337.

39 Alfarawati S, Fragouli E, Colls P, Wells D: First births after preimplantation genetic diagnosis of structural chromosome abnormalities using comparative genomic hybridization and microarray analysis. Hum Reprod 2011; 26: 1560-1574. 
40 Fiorentino F, Spizzichino L, Bono S et al: PGD for reciprocal and Robertsonian translocations using array comparative genomic hybridization. Hum Reprod 2011; 26: 1925-1935.

41 Treff NR, Northrop LE, Kasabwala K, Su J, Levy B, Scott Jr. RT: Single nucleotide polymorphism microarray-based concurrent screening of 24-chromosome aneuploidy and unbalanced translocations in preimplantation human embryos. Fertil Steril 2011; 95: $1606-1612$.

42 van Uum CM, Stevens SJ, Dreesen JC et al: SNP array-based copy number and genotype analyses for preimplantation genetic diagnosis of human unbalanced translocations. Eur J Hum Genet 2012; 20: 938-944.

Supplementary Information accompanies the paper on European Journal of Human Genetics website (http://www.nature.com/ejhg) 